\title{
Kaempferol induces apoptosis in HepG2 cells via activation of the endoplasmic reticulum stress pathway
}

\author{
HAIQING GUO $^{1 *}$, FENG REN $^{2 *}$, LI ZHANG $^{3}$, XIANGYING ZHANG ${ }^{2}$, RONGRONG YANG ${ }^{2}$, \\ BANGXIANG XIE ${ }^{2}$, ZHUO LI ${ }^{2}$, ZHONGJIE HU ${ }^{1}$, ZHONGPING DUAN $^{4}$ and JING ZHANG ${ }^{1}$ \\ ${ }^{1}$ Department of Hepatitis C and Drug-Induced Liver Disease, Beijing Youan Hospital, Capital Medical University; \\ ${ }^{2}$ Beijing Institute of Hepatology, Capital Medical University, Beijing $100069 ;{ }^{3}$ Department of Infectious Diseases, \\ The Third Affiliated Hospital of Hebei Medical University, Shijiazhuang, Hebei 050011; ${ }^{4}$ Artificial Liver Center, \\ Beijing Youan Hospital, Capital Medical University, Beijing 100069, P.R. China
}

Received April 9, 2015; Accepted December 11, 2015

DOI: $10.3892 / \mathrm{mmr} .2016 .4845$

\begin{abstract}
Kaempferol is a flavonoid compound that has gained importance due to its antitumor properties; however, the underlying mechanisms remain to be fully understood. The present study aimed to investigate the molecular mechanisms of the antitumor function of kaempferol in HepG2 hepatocellular carcinoma cells. Kaempferol was determined to reduce cell viability, increase lactate dehydrogenase activity and induce apoptosis in a concentration- and time-dependent manner in HepG2 cells. Additionally, kaempferol-induced apoptosis possibly acts via the endoplasmic reticulum (ER) stress pathway, due to the significant increase in the protein expression levels of glucose-regulated protein 78 , glucose-regulated protein 94, protein kinase R-like ER kinase, inositol-requiring enzyme $1 \alpha$, partial activating transcription factor 6 cleavage, caspase-4, C/EBP homologous protein (CHOP) and cleaved caspase-3. The pro-apoptotic activity of kaempferol was determined to be due to induction of the ER stress-CHOP pathway, as: i) ER stress was blocked by 4-phenyl butyric acid (4-PBA) pretreatment and knockdown of CHOP with small interfering RNA, which resulted in alleviation of kaempferol-induced HepG2 cell apoptosis; and ii) transfection with plasmid overexpressing $\mathrm{CHOP}$ reversed the protective effect of 4-PBA in kaempferol-induced HepG2 cells and increased the apoptotic rate. Thus, kaempferol promoted HepG2 cell apoptosis via induction of the ER stress-CHOP signaling pathway. These observations indicate that kaempferol may be used as a
\end{abstract}

Correspondence to: Dr Jing Zhang, Department of Hepatitis C and Drug-Induced Liver Disease, Beijing Youan Hospital, Capital Medical University, 8 Xitou Tiao Road, Youwai, Fengtai, Beijing 100069, P.R. China

E-mail: drzhangjing@163.com

${ }^{*}$ Contributed equally

Key words: kaempferol, apoptosis, endoplasmic reticulum stress, $\mathrm{C} / \mathrm{EBP}$ homologous protein, hepatocellular carcinoma cell potential chemopreventive treatment strategy for patients with hepatocellular carcinoma.

\section{Introduction}

Kaempferol is a flavonoid compound that is found in a variety of vegetables and fruits $(1,2)$; its chemical structure is presented in Fig. 1A. Kaempferol has been used in traditional medicine and has attracted widespread attention due to its various biological functions, including its role as an antioxidant (3), anti-inflammatory (4) and antitumor (5) compound. It has been demonstrated to have number of antitumor effects, including preventing metastasis in oral cancer (6) and inducing apoptosis of colorectal $(7)$, breast $(8,9)$ and prostate cancer $(10,11)$ and leukemia cells (12). A previous study indicated that kaempferol may induce autophagic cell death in SK-HEP-1 human hepatic cancer cells (13). However, to the best of our knowledge, the molecular mechanisms behind the antitumor effects of kaempferol on hepatocellular carcinoma (HCC) remain unknown.

The endoplasmic reticulum (ER) is involved in numerous functions, including protein synthesis, folding and secretion. Disturbances of ER function by stimuli, such as DNA damage, hypoxia, nutritional deprivation and drug toxicity, lead to the ER stress response, which subsequently triggers the unfolded protein response (UPR). As a self-protection mechanism, the UPR reduces protein synthesis and increases the expression of ER molecular chaperones glucose-regulated protein 78 (GRP78) and GRP94 to facilitate the correct folding of proteins $(14,15)$. Protein kinase RNA-like ER kinase (PERK), inositol-requiring enzyme $1 \alpha($ IRE1 $\alpha)$ and activating transcription factor 6 (ATF-6) are the three major transmembrane ER proteins. During ER stress, PERK and IRE1 $\alpha$ are activated and repress protein synthesis via the phosphorylation of the translation initiation factor, eukaryotic initiation factor $2 \alpha$. Meanwhile, ATF-6 is transported to the Golgi apparatus, where it is cleaved by Site-1 and Site- 2 proteases $(14,15)$. Collectively, these factors activate downstream signaling molecules that trigger a cascade of reactions.

Excessive and prolonged ER stress leads to cellular damage and eventually induces apoptosis. The C/EBP homologous 
protein (CHOP) is the point of convergence for the three aforementioned ER stress transducers (PERK, IRE1 $\alpha$ and ATF-6) and is also the most well-characterized factor in the transition of ER stress to apoptosis $(16,17)$. Proteins in the caspase family are the primary drivers of apoptosis. Human caspase-4 is uniquely located in the ER membrane, where it is specifically activated by ER stress. Similar to caspase-12 in mice, caspase- 4 activates caspase- 9 , in addition to other molecules such as caspase-3, eventually resulting in cell apoptosis $(18,19)$.

A previous study indicated that kaempferol induces apoptosis via the ER stress pathway in U2OS human osteosarcoma cells (20). However, to the best of our knowledge, the importance of ER stress in the antitumor activity of kaempferol in HCC has not been previously elucidated. The present study demonstrated that kaempferol triggers HepG2 apoptosis in a concentration- and time-dependent manner, and indicated that the activation of the ER stress-CHOP pathway is critical for kaempferol-induced apoptosis of HepG2 cells.

\section{Materials and methods}

Cells and cell culture. The HepG2 human hepatic cancer cell line was purchased from the American Type Culture Collection (Manassas, VA, USA). The cells were maintained in Dulbecco's modified Eagle's medium (Hyclone; GE Healthcare Life Sciences, Logan, UT, USA) supplemented with $10 \%$ fetal bovine serum (Hyclone; GE Healthcare Life Sciences) and antibiotics (100 U/ml penicillin and $100 \mathrm{mg} / \mathrm{ml}$ streptomycin; Beyotime Institute of Biotechnology, Haimen, China) and incubated at $37^{\circ} \mathrm{C}$ under a humidified atmosphere of $5 \% \mathrm{CO}_{2}$.

MTT assay for cell viability. HepG2 cells were seeded in 96-well culture plates at a density of $1 \times 10^{4}$ cells/well. Subsequent to overnight growth, the cells were incubated with 0, 5, 10, 2550 and $100 \mu \mathrm{M}$ kaempferol (Sigma-Aldrich, St. Louis, MO, USA) for $24 \mathrm{~h}$. A separate group of HepG2 cells was treated with $100 \mu \mathrm{M}$ kaempferol for time periods of 3, 6, 12 and 24 h. Kaempferol was dissolved in dimethyl sulfoxide (DMSO; Sigma-Aldrich), and the final concentration of DMSO in the culture medium was maintained at $<0.1 \%(\mathrm{v} / \mathrm{v})$. The vehicle (VE) and blank control (BC) groups were established. For the viability assay, at the end of each treatment, $200 \mu \mathrm{l}$ culture medium containing $0.5 \mathrm{mg} / \mathrm{ml} 3$-(4,5-dimethylthiazol-2-yl)-2,5-diphenyltetrazolium bromide (MTT; Amresco LLC, Solon, OH, USA) was added to each well and the mixture was incubated at $37^{\circ} \mathrm{C}$ for $4 \mathrm{~h}$. The supernatant was removed, formazan crystals were dissolved in $150 \mu \mathrm{l}$ DMSO and the absorbance was measured at $570 \mathrm{~nm}$ using a microplate reader (Multiskan MK3; Bio-Rad Laboratories, Inc., Hercules, CA, USA). To determine how kaempferol triggers apoptosis via molecular signaling pathways, the following three additional treatment groups were established: i) 4-Phenyl butyric acid (4-PBA; Sigma-Aldrich) pretreatment group, where cells were pretreated with 4-PBA at $1 \mathrm{mM}$ for $30 \mathrm{~min}$; ii) transfection group with CHOP small interfering (si) RNA; and iii) plasmid group with a CHOP overexpressing plasmid (Shanghai GenePharma Co., Ltd., Shanghai, China). HepG2 cells were then exposed to $100 \mu \mathrm{M}$ kaempferol for $24 \mathrm{~h}$, and an MTT assay was performed as abovementioned. Cell viability was calculated as follows: $\left[\left(\mathrm{A}_{\text {kaempferol treatment group }}-\mathrm{A}_{\mathrm{BC}} / \mathrm{A}_{\mathrm{VE}}-\mathrm{A}_{\mathrm{BC}}\right)\right] \times 100$, where $\mathrm{A}$ represents absorbance.

LDH activity assay. A colorimetric lactate dehydrogenase (LDH) activity assay kit (Applygen Technologies, Inc., Beijing, China) was used to quantify the level of LDH released into the supernatant from the damaged cells. This assay was performed subsequent to treatment application. The supernatants from each treatment group were collected and the assay was performed according to the manufacturer's protocol.

Flow cytometric analysis. An Annexin V-fluorescein isothiocyanate (FITC)/propidium iodide (PI) double-staining assay (Nanjing KeyGen Biotech, Co., Ltd., Nanjing, China) was used to quantify apoptosis. HepG2 cells were cultivated in $60 \mathrm{~mm}$ culture plates for $24 \mathrm{~h}$ at $37^{\circ} \mathrm{C}$. Following exposure to the indicated treatments, cells were harvested by trypsinization (Sigma-Aldrich), washed with phosphate-buffered saline (PBS), pelleted by centrifugation at $800 \mathrm{x}$ g and $4^{\circ} \mathrm{C}$ for $5 \mathrm{~min}$, and resuspended in $0.5 \mathrm{ml}$ binding buffer (Nanjing KeyGen Biotech, Co., Ltd.). The cells were incubated with $5 \mathrm{ml}$ annexin V-FITC and $5 \mathrm{ml}$ PI working solution for $15 \mathrm{~min}$ at room temperature in the dark. The samples were analyzed on a FACScan flow cytometer (BD Biosciences, Franklin Lakes, NJ, USA) using FlowJo software (version 7.6; FlowJo LLC, Ashland, OR, USA). Double staining of cells with annexin V-FITC and PI enabled the identification of different cell populations based on their staining patterns, as follows: Lower left quadrant, live cells (FITC-PI'); lower right quadrant, early apoptotic cells $\left(\mathrm{FITC}^{+} \mathrm{PI} \mathrm{I}^{-}\right)$; upper right quadrant, late apoptotic cells $\left(\mathrm{FITC}^{+} \mathrm{PI}^{+}\right)$; upper left quadrant, necrotic cells $\left(\right.$ FITC-PI $\left.^{+}\right)$.

CHOP siRNA treatment in vitro. At $24 \mathrm{~h}$ prior to transfection, HepG2 cells were prepared in 12-well culture plates. Cells were transfected with $20 \mu \mathrm{M}$ human CHOP siRNA and negative control siRNA using Lipofectamine 2000 reagent for $6 \mathrm{~h}$ according to the manufacturer's protocol (Shanghai GenePharma Co., Ltd.). CHOP siRNA sequences were designed as follows: Forward (F) 5'-GAGCUCUGAUUGACC GAAUTT-3' and reverse (R) 5'-AUUCGGUCAAUCAGAGCU CTT-3'; control siRNA, F: 5'-UGA GAG UGU CAG CAA UTT CCU-3' and R: 5'-AUC GCU UCA AAG UUA GCG CTT-3'. The transfected cells were then treated with $100 \mu \mathrm{M}$ kaempferol for $24 \mathrm{~h}$.

CHOP overexpression plasmid treatment in vitro. HepG2 cells were cultured in 12-well culture plates of $1 \mathrm{ml}$ volumes. $\mathrm{CHOP}$ overexpression plasmid (Shanghai GenePharma Co., Ltd.) and the empty vector control plasmid were transfected into HepG2 cells using Lipofectamine 2000 reagent, according to the manufacturer's protocol (Shanghai GenePharma Co., Ltd.). Following a $24 \mathrm{~h}$ transfection, HepG2 cells were treated with ER stress inhibitor 4-PBA at $1 \mathrm{mM}$ for $30 \mathrm{~min}$ at $37^{\circ} \mathrm{C}$, and cultured with $100 \mu \mathrm{M}$ kaempferol for an additional $24 \mathrm{~h}$.

Reverse transcription-quantitative polymerase chain reaction $(R T-q P C R)$ analysis. The mRNA levels of target genes were evaluated using RT-qPCR. HepG2 cells were cultured at a concentration of $1 \times 10^{5}$ cells/well in 12 -well 
A<smiles>Cc1cc(O)c2c(c1)C(c1ccccc1)=C(c1ccc(Br)cc1)C(=O)C2=O</smiles>

D
B

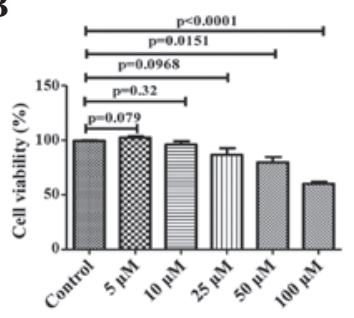

C

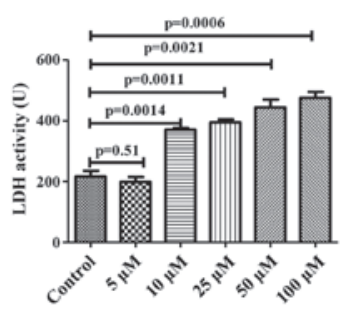

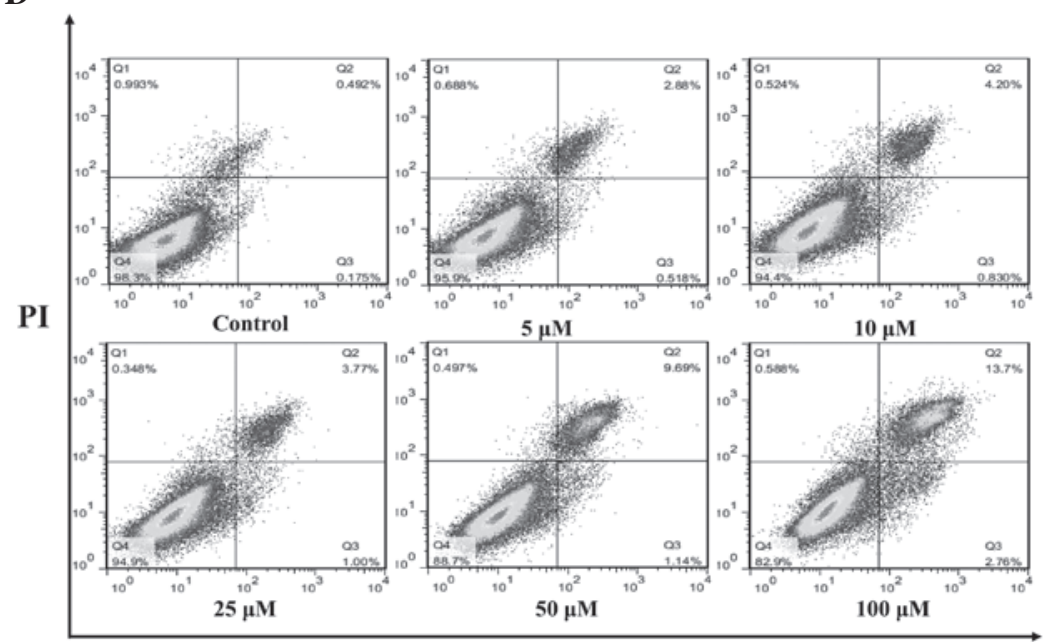

Annexin V

E

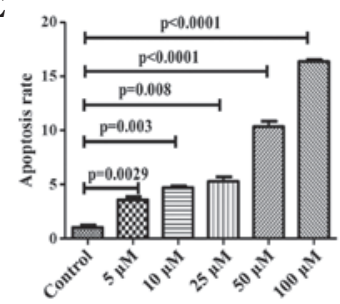

$\mathbf{F}$

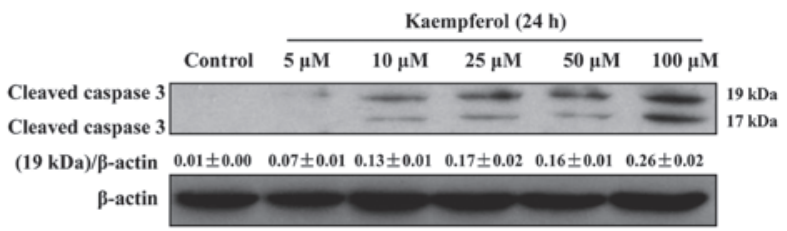

Figure 1. Dose-dependent effects of kaempferol on HepG2 cells. (A) Chemical structure of kaempferol. (B) Cell viability was reduced with higher kaempferol dosage. (C) Cell death was evaluated by determining LDH activity in the supernatant. (D) The apoptotic rate was determined using flow cytometry. (E) Quantification of apoptotic rate. (F) Expression of cleaved caspase-3 and $\beta$-actin, was determined using western blotting and quantified by densitometry. All data are expressed as the mean \pm standard deviation of at least three independent experiments. LDH, lactate dehydrogenase; PI, propidium iodide.

culture plates. Cells were cultured for $24 \mathrm{~h}$ prior to treatments. Total RNA was extracted from HepG2 cells using TRIzol reagent and then reverse-transcribed into cDNA by PrimeScript First Strand cDNA Synthesis kit (Takara Bio, Inc., Otsu, Japan), following the manufacturer's protocol. The hypoxanthine phosphoribosyl transferase (HPRT) gene was selected as an endogenous control. PCR was performed in a reaction mixture $(20 \mu \mathrm{l})$ containing $4 \mu \mathrm{l}$ cDNA, $0.4 \mu \mathrm{l}$ each primer $(10 \mu \mathrm{M}), 5.2 \mu \mathrm{l}$ diethylpyrocarbonate water and $10 \mu \mathrm{l}$ SYBR Green (Takara Bio, Inc.) using a quantitative PCR instrument (ABI Prism 7500; Applied Biosystems Inc., Waltham, MA, USA). The reaction was processed with an initial 2 min denaturation step at $50^{\circ} \mathrm{C}$, followed by $95^{\circ} \mathrm{C}$ for $5 \mathrm{~min}, 95^{\circ} \mathrm{C}$ for $15 \mathrm{sec}$, and $60^{\circ} \mathrm{C}$ for $30 \mathrm{sec}$, for $40 \mathrm{cycles}$, and then $55^{\circ} \mathrm{C}$ for $4 \mathrm{sec}$ for 41 cycles. The mRNA levels were calculated using the $2^{-\Delta \Delta \mathrm{Cq}}$ method (21). The specific primer sequences for these genes were as follows: HPRT, F 5'-TCA ACGGGGGACATAAAAGT-3' and R 5'-TGCATTGTTTTA
CCAGTGTCAA-3'; CHOP, F 5'-CCTAGCTTGGCTGAC AGAGG-3' and R 5'-CTGCTCCTTCTCCTTCATGC-3'; GPR78, F 5'-AGTGGTGCCTACCAAGAAGTCTCA-3' and R 5'-TGTCAGGGGTCTTTCACCTTCATA-3'; GPR94, F 5'-ACGTGGTCTGTTTGACGAATATGG-3' and R 5'-TAC ACGGCGCACATAGAGCTTAAT-3'.

Western blot analysis. Subsequent to the indicated treatments, cells were scraped off and washed with ice-cold PBS, and then lysed with radioimmunoprecipitation assay buffer (Sigma-Aldrich) containing a mixture of protease inhibitors. A total of $30 \mu \mathrm{g}$ of protein from each sample was separated by $12 \%$ sodium dodecyl sulfate-polyacrylamide gel (Sigma-Aldrich) electrophoresis at $80 \mathrm{v}$ for $30 \mathrm{~min}$ and $120 \mathrm{v}$ for $1 \mathrm{~h}$, and then electrotransferred onto nitrocellulose membranes (Bio-Rad Laboratories, Inc.) using the Bio-Rad Laboratories, Inc. transfer blotting system. The membranes were subsequently incubated with $5 \%$ skimmed milk in 

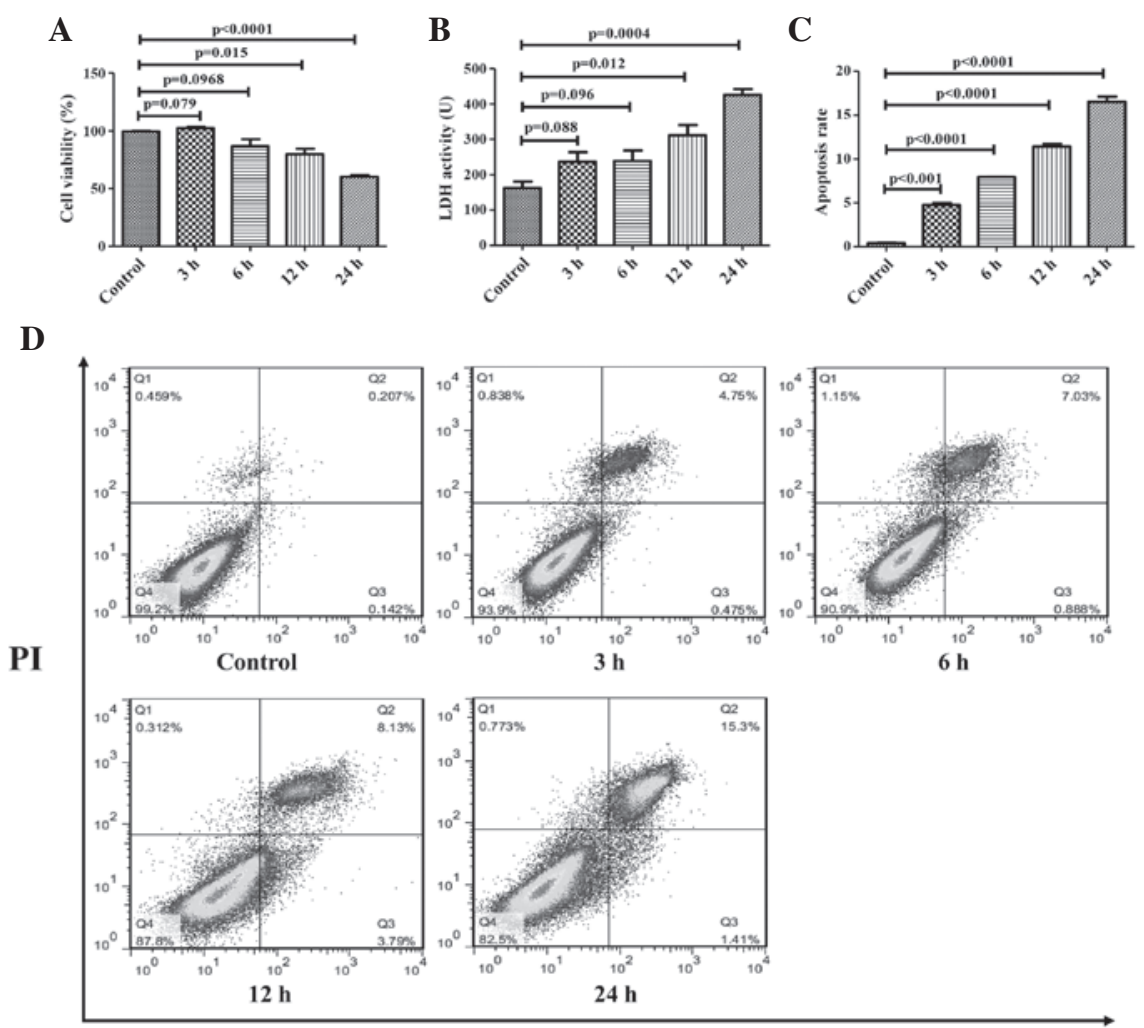

Annexin V

E

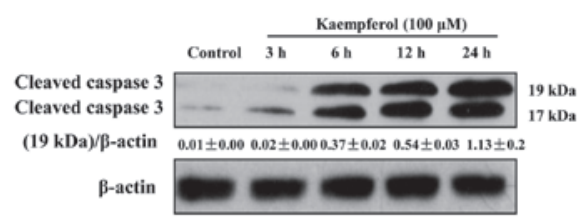

Figure 2. Time-dependent effects of kaempferol on HepG2 cells. (A) Cell viability was reduced with prolonged exposure to kaempferol. (B) Cell death was evaluated by measuring LDH activity in the supernatant. (C) The apoptotic rate was measured using flow cytometry. (D) Quantification of apoptotic rate. (E) Expression of cleaved caspase- 3 and $\beta$-actin, measured by western blotting and quantified by densitometry. All data are expressed as the mean \pm standard deviation of at least three independent experiments. LDH, lactate dehydrogenase; PI, propidium iodide.

Tris-buffered saline with Tween-20 (Beyotime Institute of Biotechnology) for $1 \mathrm{~h}$ to block nonspecific binding and then overnight with the following antibodies: Monoclonal rabbit anti-human GRP78 (1:1000; cat. no. 3177; Cell Signaling Technology, Inc., Danvers, MA, USA), monoclonal rabbit anti-human GRP94 (1:1,000; cat. no. 20292; Cell Signaling Technology, Inc.), monoclonal rabbit anti-human PERK (1:1,000; cat. no. 5683; Cell Signaling Technology, Inc.), monoclonal mouse anti-human partial ATF-6 (1:1,000; cat. no. IMG-273; Imgenex Corporation, San Diego, CA, USA), monoclonal rabbit anti-human IRE1 $\alpha$ (1:1,000; cat. no. 3294; Cell Signaling Technology, Inc.), monoclonal rabbit anti-human caspase-4 (1:1,000; cat. no. 4450; Cell Signaling Technology, Inc.), monoclonal mouse anti-human CHOP (1:1,000; cat. no. 2895; Cell Signaling Technology, Inc.), monoclonal rabbit anti-human cleaved caspase-3 (1:1,000; cat. no. 9664; Cell Signaling Technology, Inc.) and monoclonal rabbit anti-human $\beta$-actin (1:1,000; cat. no. 4970; Cell Signaling Technology, Inc.) at $4^{\circ} \mathrm{C}$, followed by incubation with monoclonal goat anti-rabbit $\operatorname{IgG}$ (1:2,000; cat. no. 14708; Cell Signaling Technology, Inc.) secondary antibody for $1 \mathrm{~h}$ at room temperature. Proteins were visualized using an enhanced chemiluminescence commercial kit (Thermo Fisher Scientific, Inc., Rockford, IL, USA). The absorbance of each well containing the protein and reagent was determined at a wavelength of $630 \mathrm{~nm}$ using a microplate reader (Multiskan MK3; Thermo Fisher Scientific, Inc.) and a protein concentration standard curve was established to calculate the concentration of each protein sample.
Statistical analysis. Statistical analyses were performed using SPSS statistical software (version 16.0: SPSS, Inc., Chicago, IL, USA). Data are expressed as the mean \pm standard deviation from a minimum of three separate experiments. Statistical comparisons between groups were performed using Student's t-test. $\mathrm{P}<0.05$ was considered to indicate a statistically significant difference.

\section{Results}

Kaempferol induces apoptosis of HepG2 cells in a dose-dependent manner. To investigate the effects of different concentrations of kaempferol on apoptosis, HepG2 cells were treated with $0-100 \mu \mathrm{M}$ kaempferol for $24 \mathrm{~h}$. Cell viability was significantly reduced at $100 \mu \mathrm{M}$ kaempferol concentration compared with the control group $(\mathrm{P}<0.0001$; Fig. 1B). Additionally, $\mathrm{LDH}$ activity $(\mathrm{P}=0.0006)$ and the rate of apoptosis significantly increased at $100 \mu \mathrm{M}$ kaempferol $(\mathrm{P}<0.0001$; Fig. 1C-E). Western blotting also indicated that kaempferol triggers expression of cleaved caspase- 3 (Fig. 1F). The results suggest that kaempferol induces apoptosis in HepG2 cells in a dose-dependent manner.

Kaempferol induces HepG2 apoptosis in a time-dependent manner. To examine whether kaempferol triggers HepG2 cells apoptosis in a time-dependent manner, HepG2 cells were treated with $100 \mu \mathrm{M}$ kaempferol for $0,3,6,12$ and $24 \mathrm{~h}$. Kaempferol gradually inhibited the proliferation of HepG2 cells 
A

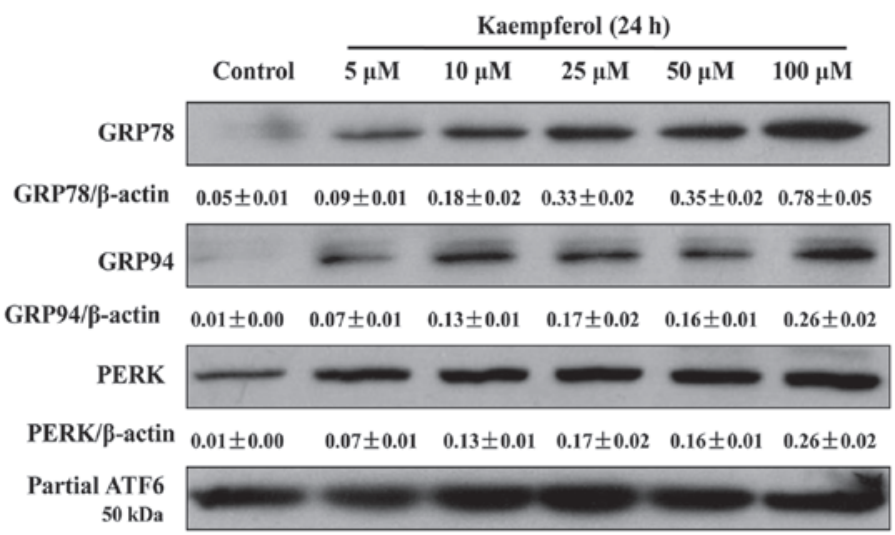

Partial ATF6/ $\beta$-actin $0.01 \pm 0.00 \quad 0.07 \pm 0.01 \quad 0.13 \pm 0.01 \quad 0.17 \pm 0.02 \quad 0.16 \pm 0.01 \quad 0.26 \pm 0.02$

IRE1 $\alpha$

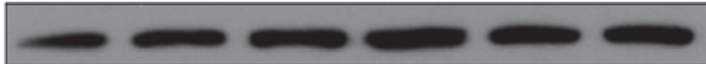

IRE1 $\alpha / \beta$-actin $0.01 \pm 0.00 \quad 0.07 \pm 0.01 \quad 0.13 \pm 0.01 \quad 0.17 \pm 0.02 \quad 0.16 \pm 0.01 \quad 0.26 \pm 0.02$

Caspase 4

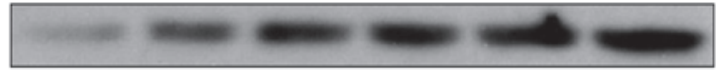

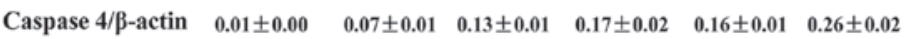

CHOP

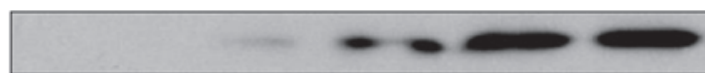

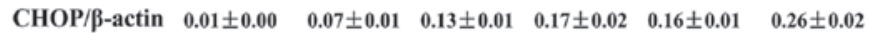

$\beta$-actin

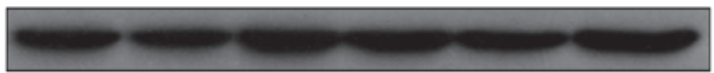

C

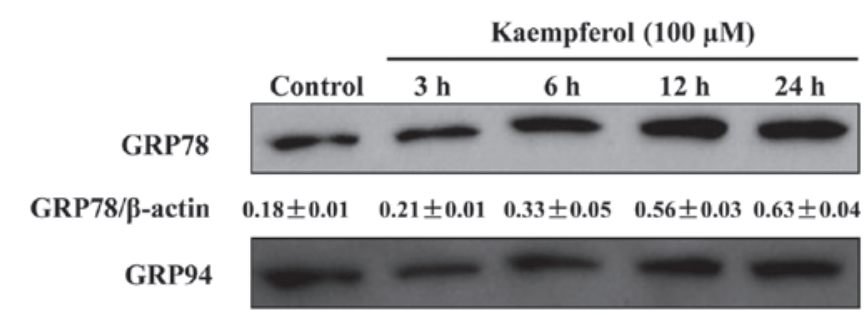

GRP94/ $\beta$-actin $\quad 0.25 \pm 0.02 \quad 0.24 \pm 0.02 \quad 0.27 \pm 0.01 \quad 0.46 \pm 0.04 \quad 0.72 \pm 0.05$

PERK

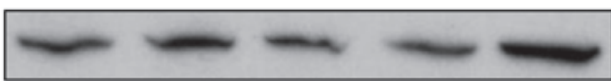

PERK/ $\beta$-actin

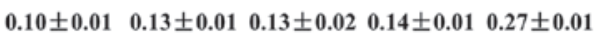

Partial ATF-6

Partial ATF6/ $\beta$-actin

IRE1a

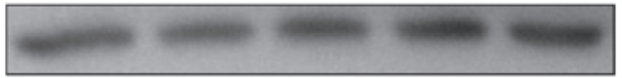

$\begin{array}{lllllllllllll}0.15 & 0.01 & 0.19 \pm 0.02 & 0.22 \pm 0.01 & 0.24 & 0.02 & 0.31 \pm 0.02\end{array}$

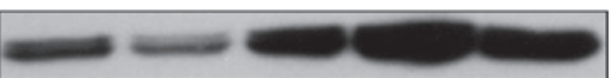

IRE1 $\alpha / \beta$-actin

$\begin{array}{lllll}0.17 \pm 0.02 & 0.13 \pm 0.01 & 1.11 \pm 0.08 & 3.10 \pm 0.2 & 2.58 \pm 0.10\end{array}$

Caspase 4

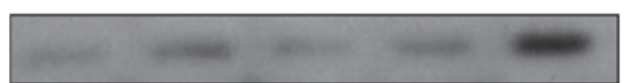

Caspase 4 / $\beta$-actin

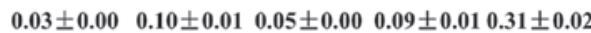

CHOP

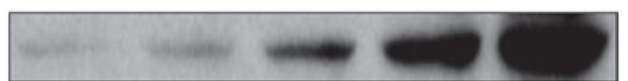

CHOP / $\beta$-actin

$0.01 \pm 0.00 \quad 0.03 \pm 0.00 \quad 0.34 \pm 0.02 \quad 1.11 \pm 0.1 \quad 3.57 \pm 0.1$

$\beta$-actin

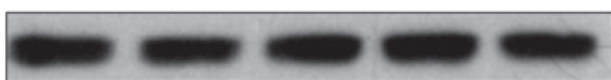

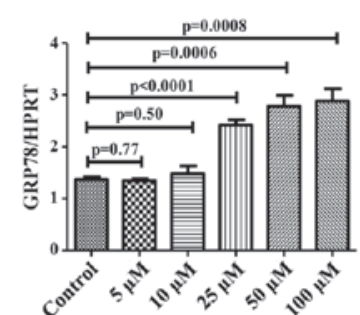
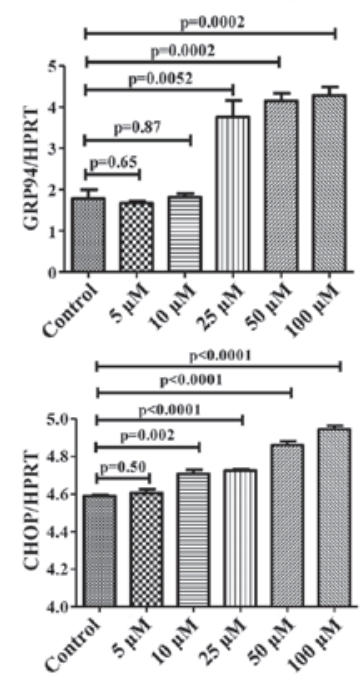

D
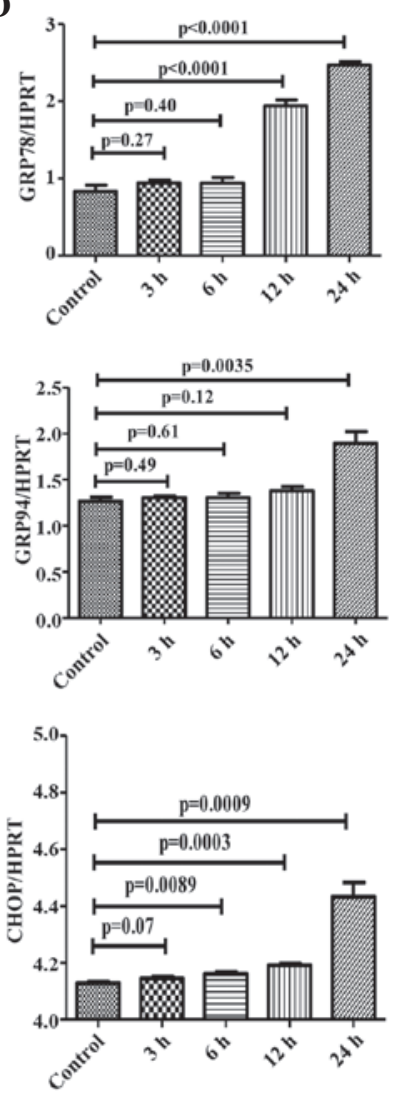

Figure 3. Dose- and time-dependent effects of kaempferol on protein and mRNA levels of endoplasmic reticulum stress markers. (A and B) For dose-dependent effects, HepG2 cells were treated in the presence or absence of different concentrations of kaempferol for $24 \mathrm{~h}$. Western blotting and RT-qPCR were performed with GRP78, GRP94, PERK, partial ATF-6, IRE1 $\alpha$, caspase-4 and CHOP antibodies. (C and D) For time-dependent effects, HepG2 cells were exposed to $100 \mu \mathrm{M}$ kaempferol for different time periods. Western blotting and RT-qPCR were performed with GRP78, GRP94, PERK, partial ATF-6, IRE1 $\alpha$, caspase- 4 and CHOP antibodies. Data are expressed as the mean \pm standard deviation of at least three independent experiments. GRP78, glucose regulated protein 78; GRP94, glucose regulated protein 94; PERK, protein kinase R-like endoplasmic reticulum kinase; ATF-6, activation transcription factor 6; IRE1 $\alpha$, inositol-requiring enzyme $1 \alpha$; CHOP, C/EBP homologous protein; RT-qPCR, reverse transcription-quantitative polymerase chain reaction; HPRT, hypoxanthine phosphoribosyl transferase. 

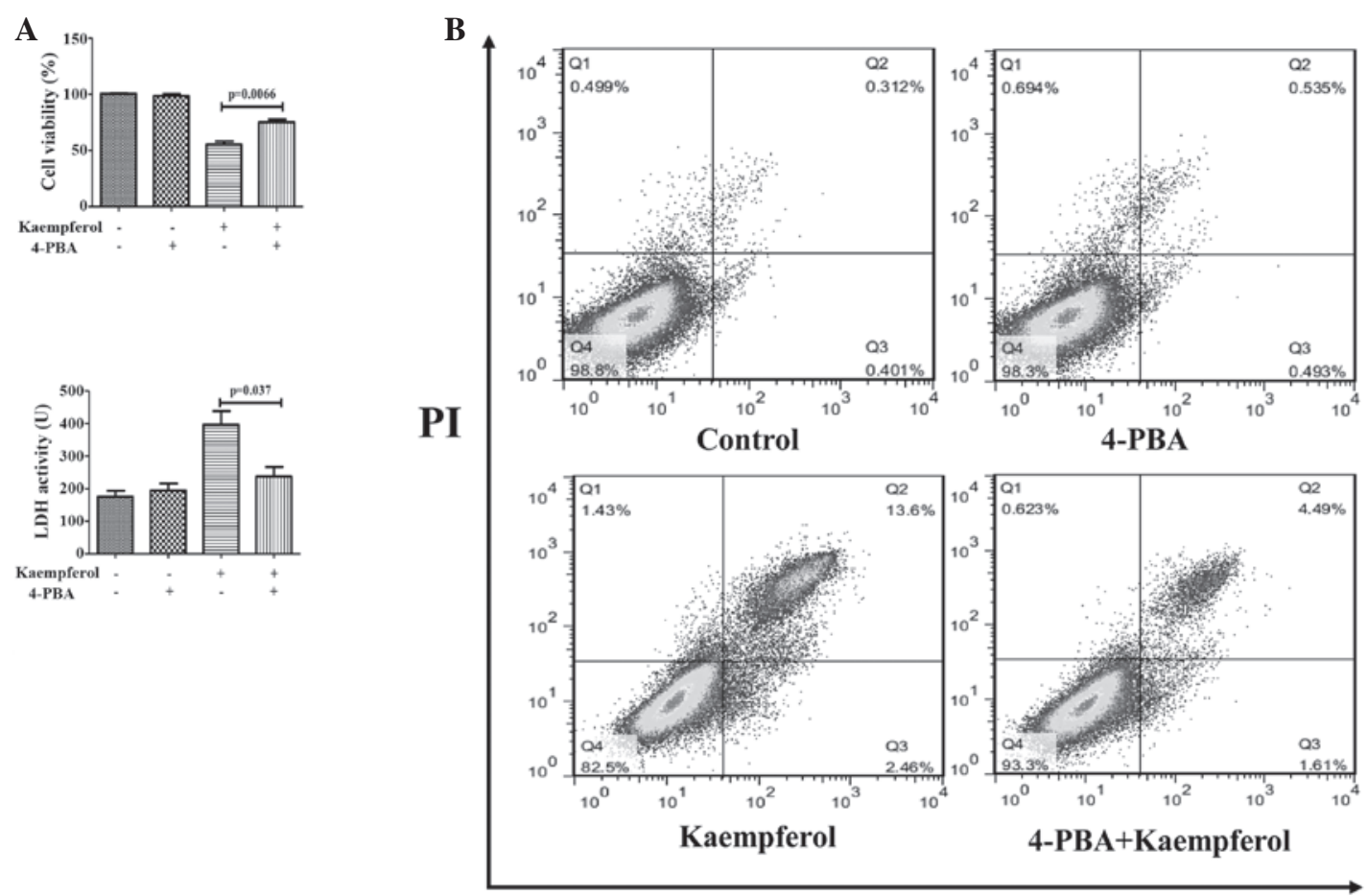

$\mathbf{C}$

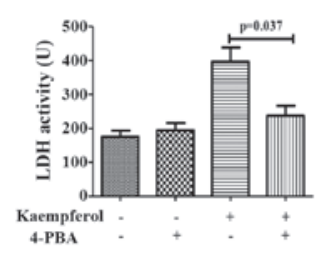

Annexin V

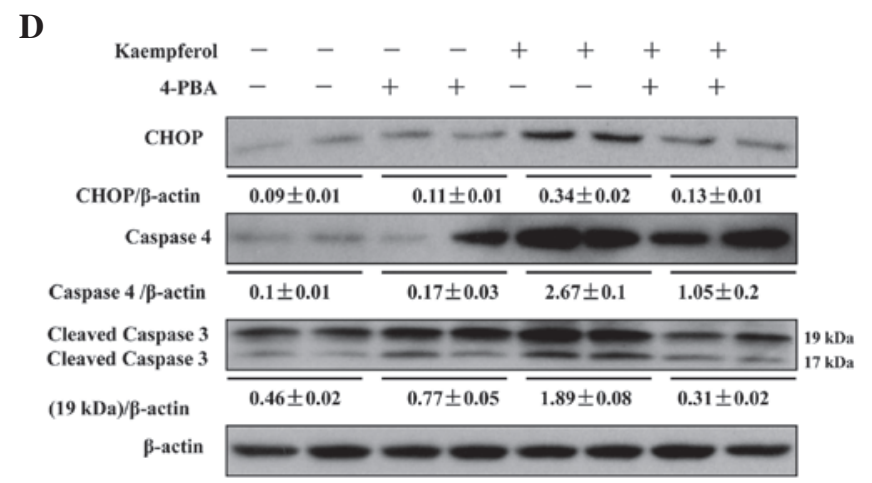

Figure 4. Endoplasmic reticulum stress inhibition by 4-PBA alleviates kaempferol-induced apoptosis in HepG2 cells. (A) Cell viability was higher and LDH activity lower in the cell group pretreated with 4-PBA compared with the kaempferol only group. (B) Apoptotic rate was determined using flow cytometry. (C) Quantification of apoptotic rate. (D) Expression levels of CHOP, caspase-4, cleaved caspase-3 and $\beta$-actin were determined using western blotting and quantified by densitometry. Data are expressed as the mean \pm standard deviation of at least three independent experiments. 4-PBA, 4-phenyl butyric acid; LDH, lactate dehydrogenase; CHOP, C/EBP homologous protein; PI, propidium iodide.

and promoted cell death between 12 and $24 \mathrm{~h}$. Following $24 \mathrm{~h}$ of $100 \mu \mathrm{M}$ kaempferol treatment, cell viability was significantly reduced $(\mathrm{P}<0.0001$; Fig. $2 \mathrm{~A})$. There was greater $\mathrm{LDH}$ activity $(\mathrm{P}<0.05$; Fig. 2B) and an increased rate of apoptosis $(\mathrm{P}<0.0001$; Fig. 2C and D) in cells exposed to kaempferol for $\geq 3 \mathrm{~h}$ compared with the control group. The expression of cleaved caspase- 3 was also elevated (Fig. 2E). These data indicate that kaempferol induces apoptosis in HepG2 cells in a time-dependent manner.

Kaempferol triggers ER stress response in a dose-and time-dependent manner. In order to explore the molecular mechanisms of kaempferol-induced apoptosis, the protein and mRNA expression levels of ER stress markers, including GRP78, GRP94, PERK, partial ATF-6, IRE1 $\alpha$, caspase-4 and CHOP were determined, using western blotting and RT-qPCR, respectively. Kaempferol treatment led to an increase of these protein and mRNA levels in a dose-and time-dependent manner (Fig. 3A and B). The protein and mRNA levels of GRP78, GRP94 and CHOP were also significantly increased with in a dose-dependant manner (Fig. 3C and D). Therefore, the results indicate that kaempferol-induced apoptosis in HepG2 cells is associated with the induction of the ER stress response.

Inhibition of ER stress alleviates kaempferol-induced HepG2 apoptosis. To confirm the role of the ER stress response as the underlying molecular mechanism of kaempferol-induced apoptosis in HepG2 cells, the cells were pretreated with the ER stress inhibitor 4-PBA to suppress ER stress. Compared with the kaempferol only treatment group, cell viability was significantly higher in the 4-PBA pretreatment group $(\mathrm{P}=0.0066$; Fig. 4A). Additionally, LDH activity 

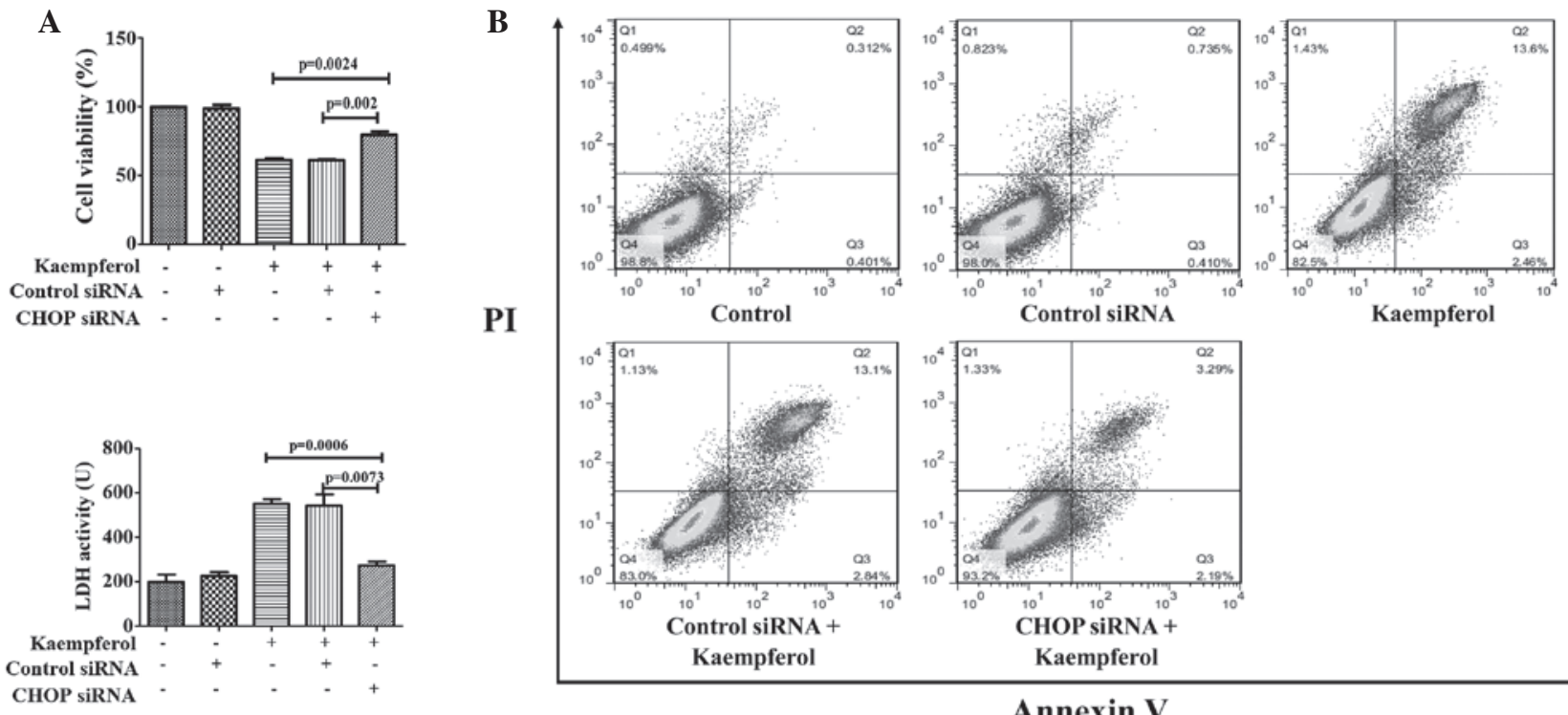

Annexin V

C

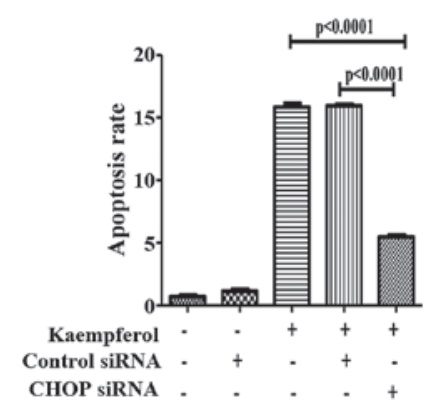

D

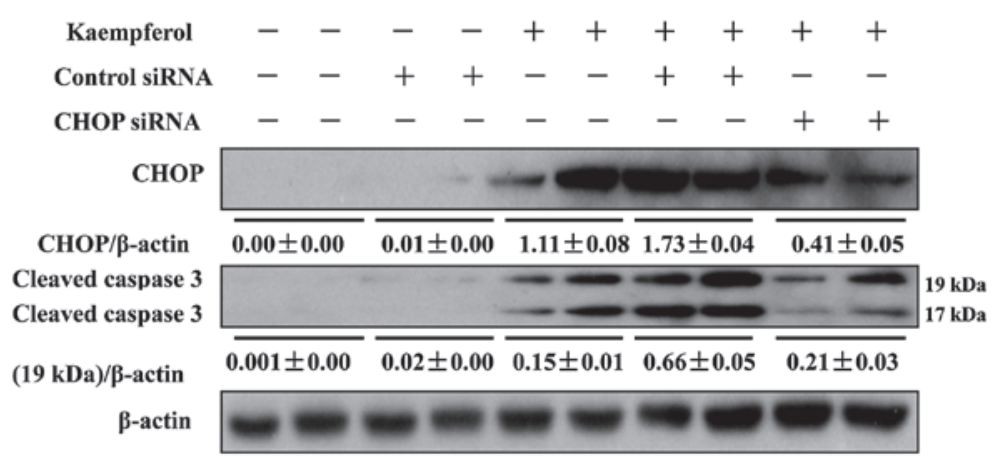

Figure 5. Knockdown of CHOP with siRNA limits kaempferol-induced apoptosis. (A) Kaempferol was found to be less effective in inducing apoptosis in cells transfected with CHOP siRNA. (B) Apoptotic rate was determined using flow cytometry. (C) Quantification of apoptotic rate. (D) Expression levels of CHOP, cleaved caspase- 3 and $\beta$-actin were determined by western blotting and quantified by densitometry. Data are expressed as the mean \pm standard deviation of at least three independent experiments. CHOP, C/EBP homologous protein; siRNA, small interfering RNA; LDH lactate dehydrogenase; PI, propidium iodide.

and apoptotic rates were lower in the 4-PBA pretreatment group compared with the kaempferol group $(\mathrm{P}=0.037$ and $\mathrm{P}=0.002$; Fig. 4A-C). Furthermore, the protein expression levels of ER stress markers were evaluated. The protein expression levels of CHOP, caspase- 4 and cleaved caspase- 3 were observed to be high in the kaempferol treatment group, whereas they were reduced subsequent to the inhibition of ER stress by 4-PBA (Fig. 4D). The results suggest that kaempferol-induced apoptosis in HepG2 cells is mediated by the ER stress response.

Kaempferol triggers ER stress to induce HepG2 apoptosis via the CHOP pathway. CHOP is an extensively-characterized factor in the transition of ER stress to apoptosis (16); therefore, its function in kaempferol-induced apoptosis in HepG2 cells was investigated by limiting its expression. Knockdown of CHOP with siRNA significantly attenuated the reduction of cell viability compared with the kaempferol only treatment group ( $\mathrm{P}=0.0024$; Fig. 5A). LDH activity $(\mathrm{P}=0.0006)$, apoptotic rate $(\mathrm{P}<0.0001)$; and protein expression levels of $\mathrm{CHOP}$ and cleaved caspase- 3 were markedly reduced in the CHOP siRNA treatment group compared with the kaempferol group (Fig. 5A-D). Following transfection with the
CHOP-overexpressing plasmid, the protein expression levels of CHOP were markedly increased, resulting in a reversal of the protective effect of 4-PBA on kaempferol-induced cell apoptosis (Fig. 6A-D). These results indicate that kaempferol promotes apoptosis of HepG2 cells via the ER stress-CHOP pathway.

\section{Discussion}

Kaempferol belongs to the flavonoid family and is found in various foods and traditional Chinese medicines $(1,2)$. As a result of its multiple uses, kaempferol has attracted widespread interest, particularly due to its antitumor properties. The present study indicates that kaempferol inhibits proliferation of HepG2 cells and promotes their apoptosis. The current study demonstrated, for the first time to the best of our knowledge, that kaempferol may induce apoptosis of hepatoma cells via the ER stress-CHOP signaling pathway.

$\mathrm{HCC}$ is characterized by high mortality rates and resistance to conventional treatments. Kaempferol has been proposed as a potential agent for HCC treatment due to its antitumor properties $(13,22-24)$. Previous studies have demonstrated that 

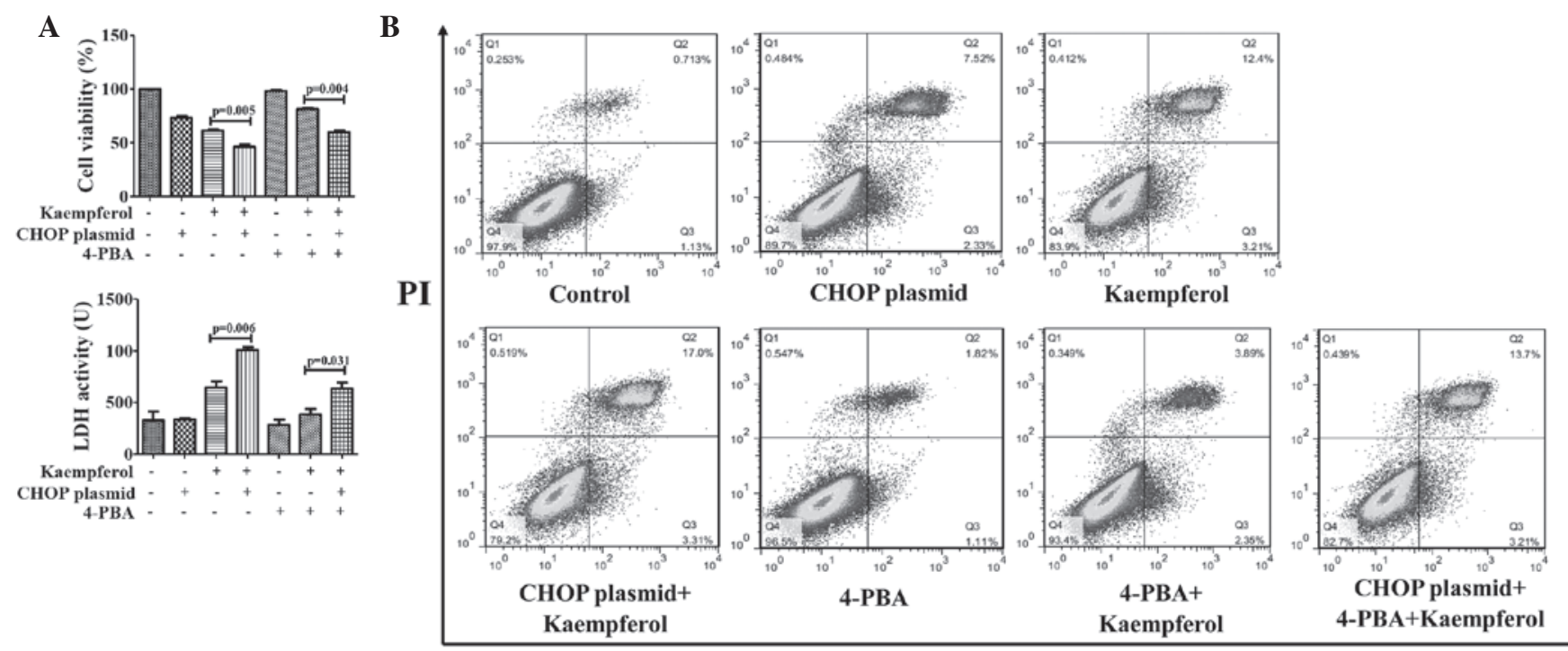

C

D

Annexin V
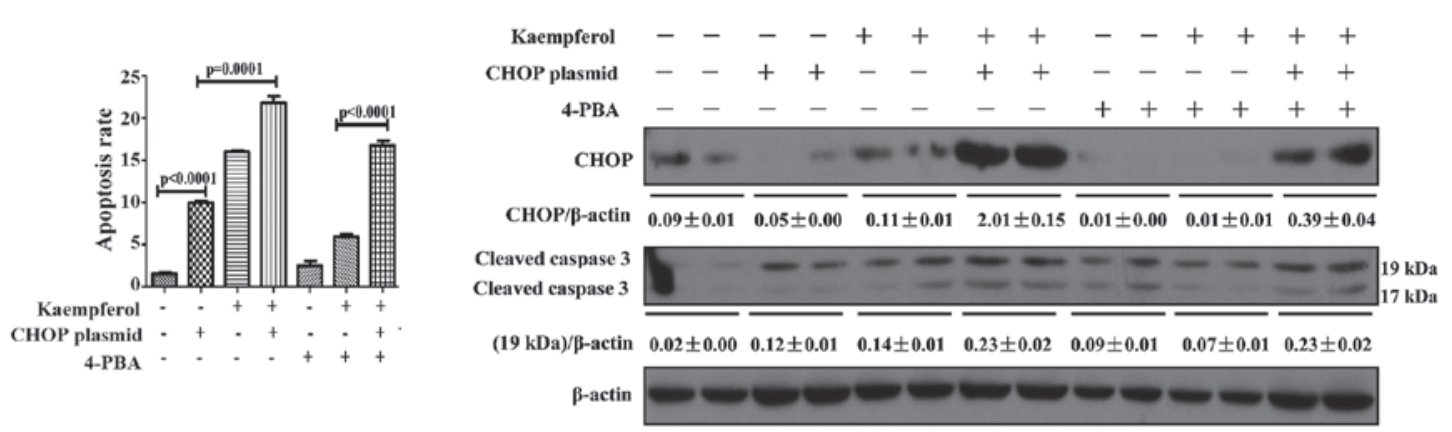

Figure 6. High expression of CHOP by transfection with the plasmid of CHOP reverses the protective effect of 4-PBA on kaempferol-induced HepG2 cell apoptosis. (A) The increased expression of CHOP led to reduced cell viability and higher LDH activity that was not alleviated by 4-PBA. (B) Apoptotic rate was measured using flow cytometry. (C) Quantification of apoptotic rate. (D) Expression of CHOP, cleaved caspase-3 and $\beta$-actin was determined by western blotting and quantified by densitometry. Data are expressed as the mean \pm standard deviation of at least three independent experiments. CHOP, C/EBP homologous protein; 4-PBA, 4-phenyl butyric acid; LDH, lactate dehydrogenase; PI, propidium iodide.

kaempferol may inhibit hypoxia-inducible factor-1 activity and induce apoptosis of Huh7 and H4IIE hepatoma cells $(22,23)$. In addition, kaempferol induces autophagic cell death in SK-HEP-1 hepatoma cells through adenosine monophosphate-activated protein kinase and protein kinase B signaling molecules (13). Kaempferol also mediated a reduction in the proliferation rate of Hep3B hepatoma cells (24). HepG2 cells were selected for the current study, as genes of interest in these cells may be easily modified (silencing or overexpression), compared with other hepatoma cells (25). In the present study, the antitumor activity of kaempferol reduced the viability of the tumor cells. Kaempferol damaged HepG2 cells, resulting in intracellular LDH release into the supernatant, which was detected as an increase in LDH activity. These results are in agreement with previous studies $(13,22-24)$, which demonstrated that kaempferol had a distinct inhibitory effect on hepatoma cellular growth. Although the suppressive effect of kaempferol on the growth of hepatoma cells may be evident from these studies, the molecular mechanisms involved remain to be fully elucidated. In current study, the effect of inhibition of proliferation of HepG2 cells was analyzed and ER stress was identified as a novel mechanism in regulating kaempferol-induced apoptosis of HCC.
The ER stress pathway is one of the three classical apoptotic pathways. It is induced in response to numerous conditions of stress $(26,27)$. The inability to properly fold proteins or remove misfolded proteins triggers the UPR to protect cells against ER stress $(28,29)$. The UPR is considered to be a cell survival mechanism; however, if ER stress cannot be alleviated, excessive and prolonged ER stress may activate caspase-4, and then caspase-3, eventually resulting in apoptosis (30). Previous studies have indicated that kaempferol induces ER stress in different cells. Kim et al (31) demonstrated that kaempferol protects cells from ischemia/reperfusion-induced cardiac damage through ER stress. Huang et al (20) demonstrated that kaempferol induces apoptosis via ER stress and a mitochondrion-dependent pathway in U2OS human osteosarcoma cells. In addition, Chandrika et al (32) also determined that kaempferol induces colon cancer cell apoptosis via the ER stress pathway. These results indicate that kaempferol-induced ER stress may be triggered via different molecular pathways in different situations. In the present study, the molecular mechanisms of kaempferol-induced apoptosis via the ER stress pathway were explored. Kaempferol induced apoptosis in HepG2 cells, as confirmed by the apoptosis rate using flow cytometry and by protein expression of cleaved caspase-3 
using western blotting. Furthermore, protein and mRNA levels of ER stress markers, GRP78, GRP94, PERK, IRE1 $\alpha$, partial ATF-6, CHOP and caspase-4, suggested that kaempferol promotes ER stress and induces ER stress-associated apoptosis in HepG2 cells. The current results indicate that promotion of excessive and prolonged ER stress is beneficial for HCC therapy.

In the current study, ER stress inhibition by 4-PBA protected HepG2 cells from kaempferol-induced apoptosis, and the protein expressions of CHOP, caspase- 4 and cleaved caspase-3 were markedly reduced in the 4-PBA pretreatment group. The contribution of CHOP to kaempferol-induced apoptosis was investigated by transfecting HepG2 cells with CHOP siRNA. The results of the current study indicated that CHOP siRNA; however not the negative control siRNA, attenuated kaempferol-induced apoptosis. However, apoptosis still occurred following treatment with 4-PBA or CHOP siRNA, suggesting that kaempferol-induced apoptosis was not completely ameliorated. This result suggests that an additional mechanism may be involved in kaempferol-induced apoptosis in HepG2 cells. In addition, to confirm the role of CHOP in kaempferol-induced ER stress, HepG2 cells were transfected with a CHOP-overexpressiing plasmid. A marked increase in the apoptosis rate and protein expression of cleaved caspase-3 in the transfected cells subsequent to administration of kaempferol was observed. The overexpression plasmid itself led to a lower apoptosis rate in transfected HepG2 cells, but not the empty vector control plasmid, strongly suggesting that high expression of CHOP promotes kaempferol-induced apoptosis. These data indicate that activation of the ER stress-CHOP pathway is one of the molecular mechanisms of kaempferol-induced HepG2 apoptosis.

In conclusion, the present results have expanded on those of previous studies and confirmed that kaempferol inhibits hepatoma cell growth. Kaempferol-induced apoptosis in HepG2 cells was at least partly mediated by ER stress; however, an additional mechanism involved in kaempferol-induced apoptosis requires further investigation. The current results also strongly suggest that the ER stress-CHOP pathway is important in kaempferol-induced apoptosis. Therefore, kaempferol is a potential therapeutic agent for HCC treatment.

\section{Acknowledgements}

The current study was supported by China National Key Project of the Twelfth Five-year Plan (grant no. 2012ZX10002005-003-003), the National Natural Science Foundation of China (grant no. 81270532), the Beijing Excellent Talents Training Fund (grant no. 2011D003034000022), the Technology Foundation for Selected Overseas Chinese Scholar, Ministry of Personnel of Beijing (2012) and Applied Research for the Clinical Characteristics of Capital (grant no. Z1211070010112167), The Special Funded Projects for 'Excellent Academic Staff' of Beijing Health Systems (grant nos. 2013-3-175 and 2011-3-082) and the Cooperation Research Project of CMU and Clinical (grant no. 13JL33).

\section{References}

1. Xiao J, Chen T and Cao H: Flavonoid glycosylation and biological benefits. Biotechnol Adv S0734-9750(14)00092-5, 2014.
2. Georgiev V, Ananga A and Tsolova V: Recent advances and uses of grape flavonoids as nutraceuticals. Nutrients 6: 391-415, 2014.

3. Huang YB, Lin MW, Chao Y, Huang CT, Tsai YH and Wu PC: Anti-oxidant activity and attenuation of bladder hyperactivity by the flavonoid compound kaempferol. Int J Urol 21: 94-98, 2014.

4. Gong JH, Shin D, Han SY, Kim JL and Kang YH: Kaempferol suppresses eosionphil infiltration and airway inflammation in airway epithelial cells and in mice with allergic asthma. J Nutr 142: 47-56, 2012.

5. Singh M, Kaur M and Silakari O: Flavones: An important scaffold for medicinal chemistry. Eur J Med Chem 84: 206-239, 2014.

6. Lin CW, Chen PN, Chen MK, Yang WE, Tang CH, Yang SF and Hsieh YS: Kaempferol reduces matrix metalloproteinase-2 expression by down-regulating ERK1/2 and the activator protein-1 signaling pathways in oral cancer cells. PLoS One 8: e80883, 2013.

7. Li W, Du B, Wang T, Wang S and Zhang J: Kaempferol induces apoptosis in human HCT116 colon cancer cells via the Ataxia-Telangiectasia Mutated-p53 pathway with the involvement of p53 Upregulated Modulator of Apoptosis. Chem Biol Interact 177: 121-127, 2009.

8. Deepa M, Sureshkumar T, Satheeshkumar PK and Priya S: Antioxidant rich Morus alba leaf extract induces apoptosis in human colon and breast cancer cells by the downregulation of nitric oxide produced by inducible nitric oxide synthase. Nutr Cancer 65: 305-310, 2013.

9. Radhika M, Ghoshal N and Chatterjee A: Comparison of effectiveness in antitumor activity between flavonoids and polyphenols of the methanolic extract of roots of Potentilla fulgens in breast cancer cells. J Complement Integr Med 9: 24, 2012.

10. Szliszka E, Zydowicz G, Janoszka B, Dobosz C, Kowalczyk-Ziomek G and Krol W: Ethanolic extract of Brazilian green propolis sensitizes prostate cancer cells to TRAIL-induced apoptosis. Int J Oncol 38: 941-953, 2011.

11. Gasmi J and Sanderson JT: Growth inhibitory, antiandrogenic, and pro-apoptotic effects of punicic acid in $\mathrm{LNCaP}$ human prostate cancer cells. J Agric Food Chem 58: 12149-12156, 2010.

12. Nandi D, Besra SE, Vedasiromoni JR, Giri VS, Rana P and Jaisankar P: Anti-leukemic activity of Wattakaka volubilis leaf extract against human myeloid leukemia cell lines. J Ethnopharmacol 144: 466-473, 2012.

13. Huang WW, Tsai SC, Peng SF, Lin MW, Chiang JH, Chiu YJ, Fushiya S, Tseng MT and Yang JS: Kaempferol induces autophagy through AMPK and AKT signaling molecules and causes G2/M arrest via downregulation of CDK1/cyclin B in SK-HEP-1 human hepatic cancer cells. Int J Oncol 42: 2069-2077, 2013.

14. Chen S, Xuan J, Couch L,Iyer A, Wu Y, Li QZ and Guo L: Sertraline induces endoplasmic reticulum stress in hepatic cells. Toxicology 322: 78-88, 2014.

15. Zhang Y, Xue R, Zhang Z, Yang X and Shi H: Palmitic and linoleic acids induce ER stress and apoptosis in hepatoma cells. Lipids Health Dis 11: 1, 2012.

16. Marhfour I, Lopez XM, Lefkaditis D, Salmon I, Allagnat F, Richardson SJ, Morgan NG and Eizirik DL: Expression of endoplasmic reticulum stress markers in the islets of patients with type 1 diabetes. Diabetologia 55: 2417-2420, 2012.

17. McCloy RA, Shelley EJ, Roberts CG, Boslem E, Biden TJ, Nicholson RI, Gee JM, Sutherland RL, Musgrove EA, Burgess A and Butt AJ: Role of endoplasmic reticulum stress induction by the plant toxin, persin, in overcoming resistance to the apoptotic effects of tamoxifen in human breast cancer cells. Br J Cancer 109: 3034-3041, 2013.

18. Xie Y, Tao X, Cheng Z, Guan Q, Yang W and Zhu Y: Discrepancy of uterine leiomyoma and myometrium to hypoxia-induced endoplasmic reticulum stress after uterine occlusion therapy accounts for therapeutic effect. Arch Gynecol Obstet 289: 1039-1045, 2014

19. Gorman AM, Healy SJ, Jäger R and Samali A: Stress management at the ER: Regulators of ER stress-induced apoptosis. Pharmacol Ther 134: 306-316, 2012.

20. Huang WW, Chiu YJ, Fan MJ, Lu HF, Yeh HF, Li KH, Chen PY, Chung JG and Yang JS: Kaempferol induced apoptosis via endoplasmic reticulum stress and mitochondria-dependent pathway in human osteosarcoma U-2 OS cells. Mol Nutr Food Res 54: 1585-1595, 2010.

21. Livak KJ and Schmittgen TD: Analysis of relative gene expression data using real-time quantitative PCR and the 2(-Delta Delta C(T)) Method. Methods 25: 402-408, 2001.

22. Mylonis I, Lakka A, Tsakalof A and Simos G: The dietary flavonoid kaempferol effectively inhibits HIF-1 activity and hepatoma cancer cell viability under hypoxic conditions. Biochem Biophys Res Commun 398: 74-78, 2010. 
23. Niering P, Michels G, Wätjen W, Ohler S, Steffan B, Chovolou Y, Kampkötter A, Proksch P and Kahl R: Protective and detrimental effects of kaempferol in rat H4IIE cells: Implication of oxidative stress and apoptosis. Toxicol Appl Pharmacol 209: 114-122, 2005.

24. Berger A, Venturelli S, Kallnischkies M, Böcker A, Busch C, Weiland T, Noor S, Leischner C, Weiss TS, Lauer UM, et al: Kaempferol, a new nutrition-derived pan-inhibitor of human histone deacetylases. J Nutr Biochem 24: 977-985, 2013.

25. Guo L, Dial S, Shi L, Branham W, Liu J, Fang JL, Green B, Deng H, Kaput J and Ning B: Similarities and differences in the expression of drug-metabolizing enzymes between human hepatic cell lines and primary human hepatocytes. Drug Metab Dispos 39: 528-538, 2011.

26. Ozcan U, Yilmaz E, Ozcan L, Furuhashi M, Vaillancourt E, Smith RO, Görgün CZ and Hotamisligil GS: Chemical chaperones reduce ER stress and restore glucose homeostasis in a mouse model of type 2 diabetes. Science 313: 1137-1140, 2006.

27. Zode GS, Kuehn MH, Nishimura DY,Searby CC, Mohan K, Grozdanic SD, Bugge K, Anderson MG, Clark AF, Stone EM and Sheffield VC: Reduction of ER stress via a chemical chaperone prevents disease phenotypes in a mouse model of primary open angle glaucoma. J Clin Invest 121: 3542-3553, 2011.
28. Ron D and Walter P: Signal integration in the endoplasmic reticulum unfolded protein response. Nat Rev Mol Cell Biol 8: 519-529, 2007.

29. Xu C, Bailly-Maitre B and Reed JC: Endoplasmic reticulum stress: Cell life and death decisions. J Clin Invest 115: 2656-2664, 2005.

30. Kim I, Xu W and Reed JC: Cell death and endoplasmic reticulum stress: Disease relevance and therapeutic opportunities. Nat Rev Drug Discov 7: 1013-1030, 2008.

31. Kim DS, Ha KC, Kwon DY, Kim MS, Kim HR, Chae SW and Chae HJ: Kaempferol protects ischemia/reperfusion-induced cardiac damage through the regulation of endoplasmic reticulum stress. Immunopharmacol Immunotoxicol 30: 257-270, 2008

32. Chandrika BB, Maney SK, Lekshmi SU, Joseph J, Seervi M, K S P and T R S: Bax deficiency mediated drug resistance can be reversed by endoplasmic reticulum stress induced death signaling. Biochem Pharmacol 79: 1589-1599, 2010. 